\title{
Mechanical and electronic properties of vanadium oxide nanotubes
}

\author{
B. Sipos, ${ }^{1}$ M. Duchamp, ${ }^{1}$ A. Magrez, ${ }^{1}$ L. Forró, ${ }^{1, a)}$ N. Barišić, ${ }^{2}$ A. Kis, ${ }^{3}$ J. W. Seo, ${ }^{4, b)}$ \\ F. Bieri, ${ }^{5}$ F. Krumeich, ${ }^{5}$ R. Nesper, ${ }^{5,6}$ and G. R. Patzke ${ }^{7}$ \\ ${ }^{1}$ Institute of Physics of Complex Matter, Ecole Polytechnique Fédérale de Lausanne, CH-1015 Lausanne, \\ Switzerland \\ ${ }^{2}$ 1. Physikalisches Institut, Universität Stuttgart, D-70550 Stuttgart, Germany \\ ${ }^{3}$ Institute of Electrical Engineering, Ecole Polytechnique Fédérale de Lausanne, CH-1015 Lausanne, \\ Switzerland \\ ${ }^{4}$ Department of Metallurgy and Materials Engineering, Katholieke Universiteit Leuven, B-3001 Heverlee, \\ Belgium \\ ${ }^{5}$ Laboratory of Inorganic Chemistry, ETH Zürich, CH-8093 Zürich, Switzerland \\ ${ }^{6}$ Collegium Helveticum, Common Institution of ETH and University of Zürich, CH-8093 Zürich, Switzerland \\ ${ }^{7}$ Institute of Inorganic Chemistry, University of Zürich, CH-8006 Zürich, Switzerland
}

(Received 24 November 2008; accepted 17 February 2009; published online 14 April 2009)

\begin{abstract}
Vanadium oxide nanotubes with a diameter of 20-100 $\mathrm{nm}$ and an aspect ratio of 50-100 were synthesized by hydrothermal method. Young's modulus of 20-80 GPa was obtained by bending measurements of individual nanotubes using an atomic force microscope. Electrical resistivity and thermopower measurements on a large assembly of nanotubes determined the characteristic energies required to put a charge into a polaronic site $\left(\Delta E_{g}=0.20 \mathrm{eV}\right)$ and to extract and propagate the polaron between neighboring sites $\left(\Delta E_{p}=0.09 \mathrm{eV}\right)$. (C) 2009 American Institute of Physics.
\end{abstract} [DOI: $10.1063 / 1.3103280$ ]

\section{INTRODUCTION}

Since the discovery of carbon nanotubes, quasi-onedimensional materials have attracted great interest because of their remarkable properties and a wide range of significant technological implications. ${ }^{1}$ Unfortunately, carbon nanotubes exist in different chiralities and diameters, corresponding to metallic or semiconducting tubes. Although some control over the tube diameter has been achieved during synthesis on patterned substrates, the state of the art carbon nanotube growth does not yet allow obtaining tubes of one specific type. Therefore, nanostructures that are more uniform in structure and in electronic properties are of importance. Possible alternatives include nanotubes, nanowires, nanorods, and nanoribbons of various elements and compounds that have been synthesized meanwhile. ${ }^{2}$ Especially, nanotubes of transition metal oxides represent a unique class of materials because of the variable oxidation state of the transition metal, which is connected with interesting electrochemical and physical properties. ${ }^{3}$

Vanadium oxide $\left(\mathrm{VO}_{x}\right)$ is a system that forms nanotubes as well as nanowires. ${ }^{4}$ The tube walls consist of bent $\mathrm{VO}_{x}$ layers between which organic molecules are embedded. The $\mathrm{VO}_{x}$ layers are crystalline with planar unit cell of $\mathrm{V}_{7} \mathrm{O}_{16}$ (Ref. 5) containing two sheets of edge-sharing pentagonal pyramids coupled in zigzag chains. These sheets are connected via $\mathrm{VO}_{4}$ tetrahedra. In this structure, mixed valency of $\mathrm{V}$ ions occurs $\left(\mathrm{V}^{4+}\right.$ and $\left.\mathrm{V}^{5+}\right)$ and contributes to the electronic conductivity. The relative $\mathrm{V}^{4+}$ content was calculated to about $46 \%$ yielding a valency of about $4.54+.{ }^{4}$ From x-ray photoelectron spectroscopy and electron energy loss spec-

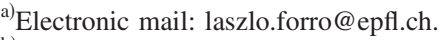

${ }^{b)}$ Electronic mail: maria.seo@mtm.kuleuven.be.
}

troscopy (EELS) measurements a nonzero occupation of the $\mathrm{V} 3 d$ shell was observed, ${ }^{6}$ and the averaged vanadium valency was determined to be $4.4+$.

Recently, nanotubes of $\mathrm{VO}_{x}$ have attracted much attention because Krusin-Elbaum et $a .^{7}$ reported that the asformed $\mathrm{VO}_{x}$ nanotubes can be transformed from spinfrustrated semiconductor to ferromagnet by doping with either electrons $(\mathrm{Li})$ or holes $(\mathrm{I})$, yielding room-temperature ferromagnetic material for spintronic applications. Nevertheless, pristine $\mathrm{VO}_{x}$ nanotubes are also attractive for lithium batteries ${ }^{8}$ and thermochromic applications; ${ }^{9}$ they are good gas sensors with extremely high sensitivity and selectivity, ${ }^{10}$ optical limiters ${ }^{11}$ in the visible and infrared spectral ranges, etc. The simple and low-cost synthesis procedure involving soft chemistry is also an advantage for this material. ${ }^{12}$ These features show that $\mathrm{VO}_{x}$ is a promising functional material; hence it is important to explore its physical properties in both forms, i.e., the properties of individual tubes and that of large arrays. Therefore, in this contribution, we report on the mechanical and electronic properties of $\mathrm{VO}_{x}$ nanotubes.

\section{EXPERIMENTAL DETAILS}

The $\mathrm{VO}_{x}$ nanotubes were prepared using a hydrothermal procedure described in detail in previous works. ${ }^{3,4}$ As $\mathrm{V}_{2} \mathrm{O}_{5}$ is a nonstoichiometric compound because of oxygen vacancies, we use the notation $\mathrm{VO}_{x}$, but $\mathrm{V}_{2} \mathrm{O}_{5-\delta}$ would be equally good. Figure 1 shows a representative transmission electron micrograph of $\mathrm{VO}_{x}$ nanotubes used in this study. The length of the tubes varied between 1 and $10 \mu \mathrm{m}$ while the diameter was in the $20-100 \mathrm{~nm}$ range. As found by transmission electron microscopy (TEM), the tubes were open ended. It has to be mentioned that the $\mathrm{VO}_{x}$ tubes are not perfect cylinders but scrolls made from a single $\mathrm{VO}_{x}$ sheet. Detailed TEM investigations revealed that $\mathrm{VO}_{x}$ forms concentric layers as well 


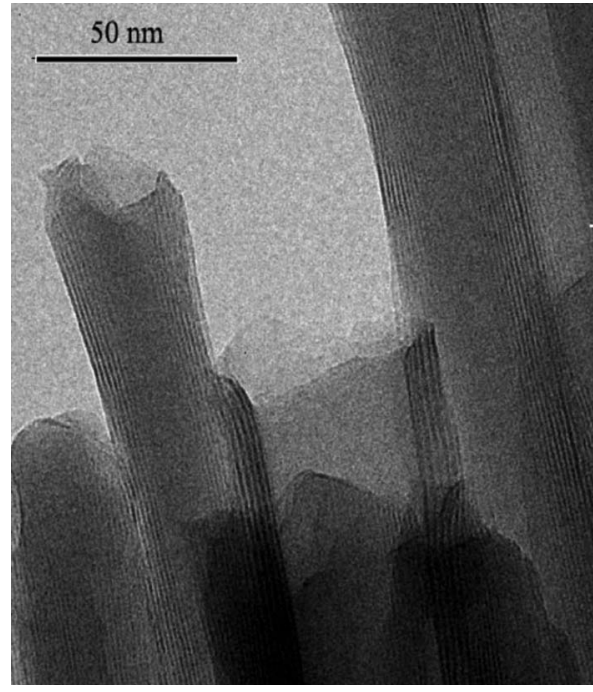

FIG. 1. Representative TEM image of $\mathrm{VO}_{x}$ nanotubes used in the present study. The walls around the empty core are visible as well as that the tubes are open ended.

as scrolls. ${ }^{4,13}$ Most tubes are disordered variants of the pure forms. Actually, the synthesis involves the use of amines without which no tubes are formed. The length of the $\mathrm{VO}_{x}$ tubes increases with the alkyl chain of the amine. It was found that amine molecules are intercalated within the scroll structure in-between the tubes. This structural characterization corroborates with previous studies. ${ }^{6,11}$

For the transport and thermoelectric power (Seebeckcoefficient) measurements, we have produced a $\mathrm{VO}_{x}$ nanotube film by drawing a tube suspension through a $0.2 \mu \mathrm{m}$ pore ceramic filter, which left a uniform grayish/black deposit on the filter. The residue of randomly oriented $\mathrm{VO}_{x}$ nanotubes was about $1 \mathrm{~mm}$ thick and it was subsequently vacuum dried. Rectangular-shape samples with a typical size of $8.0 \times 5.0 \mathrm{~mm}^{2}$ were cut out from the deposit and used for measurements.

The resistivity was measured in a conventional fourprobe configuration. Gold-wire electrodes were attached to the sample by using a silver paste. Its temperature dependence was measured in the 160-800 K temperature range. To perform the thermoelectric power measurement the sample was mounted on a ceramic sample holder, whereupon miniaturized heaters were attached to both ends for generating a temperature gradient which was measured with a differential K-type thermocouple. A typical temperature difference was 1 $\mathrm{K}$.

For the measurement of the elastic property, individual $\mathrm{VO}_{x}$ nanowires were used. We applied the technique developed in our group by Salvetat et al. ${ }^{14}$ for the measurements of carbon nanotubes. The nanotubes were dispersed in ethanol by sonication, and their dispersion was placed over alumina or $\mathrm{Si}_{3} \mathrm{~N}_{4}$ membranes. Occasionally nanotubes lie over a hole with a short section of their entire length, whereas the major part of the nanotube is still in contact with the membrane surface and therefore can be considered as clamped by van der Waals forces [see Fig. 2(a)]. By means of an atomic force microscope (AFM), changes in the vertical deflection $(\delta)$ are measured as a function of the nominal applied force (a)

(b)
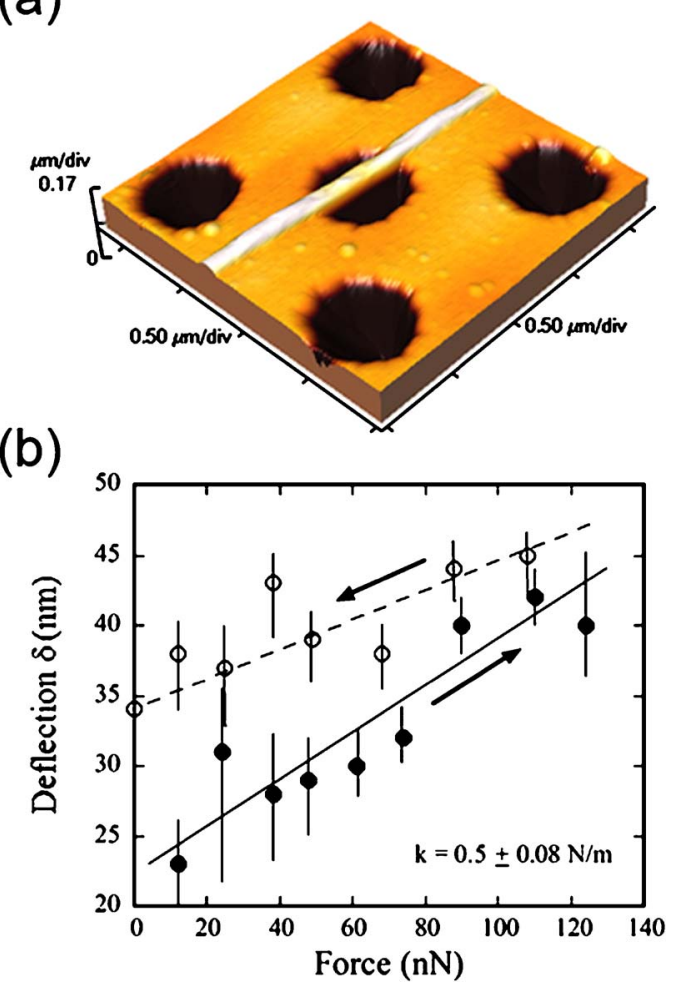

FIG. 2. (Color online) (a) AFM image of a $\mathrm{VO}_{x}$ nanotube adhered to the $\mathrm{Si}_{3} \mathrm{~N}_{4}$ membrane with a portion bridging a pore of $500 \mathrm{~nm}$ of the membrane. (b) Nanotube deflection vs applied force for loading (full dots) and unloading (empty dots). The residual deflection is due to permanent deformation. The spring constant $k$ of the cantilever is also given.

$(F)$, which is inversely proportional to Young's modulus of the nanotube. Using simple beam mechanics, Young's modulus $(E)$ is expressed as $E=F L^{3} / \delta \alpha I$, where $\alpha=192$ for a clamped beam, $F$ is the applied force varying from image to image, $I=\pi\left(D^{4}-D_{i}^{4}\right) / 64$ is the moment of inertia, and $D_{i}$ is the inner radius of a tube. The height of the nanotube is derived from a part that lies on the flat membrane surface and is set equal to the diameter $D$. For the suspended length $L$ we take the pore width on sites just next to the nanotube. The systematic error mainly originates from the uncertainty in $L$. Note that the lack of knowing the inner diameter $\left(D_{i}\right)$ of the measured tube introduces a minor error in the absolute value due to its fourth power in the expression of $E$. Calculation of $E$ is based on the assumption that the nanotube is perfectly clamped to the membrane. Adhesion depends on the real contact surface between the nanotube and the substrate, and if the contact surface is small (either due to the bent structure of the tube or to a nonflat surface) the nanotube can move. This situation can be screened by taking subsequent images of the nanotube/membrane assembly. In the data presented here, the nanotubes were firmly clamped to the surface.

\section{RESULTS AND DISCUSSION}

A typical force-deflection curve for loading and unloading is shown in Fig. 2(b). Every point corresponds to a scanning of the nanotube/membrane in contact mode with a constant force. On subsequent scans the position of the nanotube 
TABLE I. Summary of the AFM measurements.

\begin{tabular}{ccccc}
\hline \hline Tube No. & $\begin{array}{c}\text { Diameter } \\
(\mathrm{nm})\end{array}$ & $\begin{array}{c}\text { Suspended length } \\
(\mathrm{nm})\end{array}$ & $\begin{array}{c}\text { Young's modulus } \\
(\mathrm{GPa})\end{array}$ & Substrate \\
\hline 1 & 18.5 & 316 & $80 \pm 40$ & $\mathrm{Al}_{2} \mathrm{O}_{3}$ \\
2 & 36 & 666 & $30 \pm 15$ & $\mathrm{Si}_{3} \mathrm{~N}_{4}$ \\
3 & 36 & 700 & $20 \pm 10$ & $\mathrm{Si}_{3} \mathrm{~N}_{4}$ \\
\hline \hline
\end{tabular}

was the same (thus, no sliding on the membrane surface); only its deflection increased over the hole. The slope of $\delta$ versus $F$ is inversely proportional to Young's modulus of the nanotube (see the expression above). In many cases the slope of the first loading curve is significantly higher than the unloading one. In our interpretation this is due to presence of the amine groups in-between the layers. These organic molecules render the mechanical response softer until they are squeezed by the increasing load. Coming back from the highest load, the nanotube gives a more intrinsic, higher modulus. Due to this phenomenon, the measured values of Young's modulus span the 20-80 GPa range but we believe that the higher values are more intrinsic to the $\mathrm{VO}_{x}$ layers. Selected measurements with all the important parameters are listed in Table I.

How do these values compare to Young's modulus reported on other forms of vanadium pentoxide? The most common phase is the $\alpha-\mathrm{V}_{2} \mathrm{O}_{5}$ which is built up from $\mathrm{VO}_{5}$ square pyramids shearing edges and corners which build up layers. The $\mathrm{V}_{2} \mathrm{O}_{5}$ layers are held together by weak, van der Waals type $\mathrm{V}-\mathrm{O}$ interactions. Due to this architecture of the lattice, the mechanical response is very anisotropic. The modulus in one direction could be as high as $220 \mathrm{GPa}$, while it cleaves ${ }^{15}$ very easily along the (001) planes at that point that $\mathrm{V}_{2} \mathrm{O}_{5}$ is foreseen to be a promising solid lubricant. ${ }^{16}$ This is very similar to the case of graphite, which has a high in-plane modulus but low shear modulus between the planes. By rolling up the graphite sheets as carbon nanotubes, the low shear modulus is eliminated. A similar effect is expected for $\mathrm{VO}_{x}$ tubes: Rolling up the $\mathrm{V}_{2} \mathrm{O}_{5}$ layers one should approach the high modulus measured for single crystals. The fact that the measured values are low is due to defects in the tubes. Already for single crystals Jachmann and Hucho ${ }^{15}$ showed that the elastic modulus is very sensitive to the stoichiometry of the compound. Fateh et al. ${ }^{17}$ reported that with the increase in the crystallinity of the thin films of $\mathrm{V}_{2} \mathrm{O}_{5}$, Young's modulus can increase from 80 to $130 \mathrm{GPa}$.

Young's modulus of the $\mathrm{VO}_{x}$ nanotubes presented here is far from that of single crystals or well-ordered thin films. Although the structure is crystalline at a local scale, it is less ordered on a long range as TEM analysis revealed. This could be due to the low temperature synthesis of the nanotubes and/or to the oxygen deficiency in the structure. These combined effects make the overall mechanical response weak. In many nanostructures (nanotubes and nanowires) significantly lower Young's modulus compared to the bulk value has been observed, originating from different levels of defects and variance in their synthesis conditions. In the case of carbon nanotubes, the value of Young's modulus is assumed to be around $1 \mathrm{TPa}$ for multi- and single-walled car- bon nanotubes grown by arc-discharge and laser ablation methods whereas carbon nanotubes produced by catalytic chemical vapor deposition show Young's modulus lower than $100 \mathrm{GPa}^{18,19}$ Similar effects have been observed for $\mathrm{ZnO}$ nanowires with Young's modulus of about $100 \mathrm{GPa}$, which is $30 \%$ lower than the bulk value. ${ }^{20}$ For $\mathrm{VO}_{x}$ nanotubes, although $p$ and $n$ doping may render the nanotubes ferromagnetic ${ }^{7}$ and make them very promising for spin dependent scanning probe applications, the structural perfectness of these tubes has to be improved. In particular, for Li-doped nanotubes, Hellmann et al. ${ }^{21}$ recently indicated that the structural instability is a major concern. Although the charge transfer upon doping could be confirmed by EELS, the tubes were strongly electron beam sensitive, indicating a metastable structure. Moreover, the Li doping was found to disappear upon short exposure to air. This phenomenon was explained by the preferential diffusion of $\mathrm{Li}$ ions to the sample surface and by the formation of Li oxide or hydroxide.

Extended structural defects can influence the dc electrical conductivity as well. Indeed, resistivity measurements on individual $\mathrm{VO}_{x}$ nanotubes gave a substantial scattering in the absolute value at $300 \mathrm{~K}$ in the range of $10^{3}-10^{5} \Omega \mathrm{cm}$ (not shown). This large dispersion might be due to extended defects in the structure of the tubes, maybe related to the amine groups in-between the layer, and/or to the difference in oxygen off-stoichiometry of different nanotubes. In many applications, a large assembly of nanotubes is more attractive than individual nanotubes, in particular, because of the easier handling. Transport measurement on such assembly shows higher reproducibility. It seems that the more conducting tubes (or parts of the tubes) form a good percolative network. The room-temperature value of resistivity is in the range of $200 \Omega \mathrm{cm}$.

Band structure calculations ${ }^{22}$ and optical absorption measurements ${ }^{23}$ of $\mathrm{V}_{2} \mathrm{O}_{5}$ revealed a band-gap energy of 2.4$2.7 \mathrm{eV}$. However, when one plots the logarithm of resistivity as $1 / T$, the activation energy is much less than that; it is in the $0.2-0.4 \mathrm{eV}$ range, pointing toward a low-lying impurity level. ${ }^{24,25}$ Indeed, it is considered that vanadium pentoxide (in any forms) is always oxygen deficient, and oxygen vacancies are contributing to the electronic conductivity in the following way: The vacancies are compensated by excess electrons located on vanadium sites, reducing $\mathrm{V}^{5+}$ to $\mathrm{V}^{4+}$. In this way, the unpaired electron is localized on two vanadium sites associated with an oxygen vacancy. As electron spin resonance (ESR) and dc conductivity measurements on $\mathrm{V}_{2} \mathrm{O}_{5}$ single crystals revealed, the localized charge deforms the lattice in its vicinity leading to the formation of small polarons. ${ }^{26}$ These measurements revealed an activation energy of about $0.1 \mathrm{eV}$ at low temperature (below $160 \mathrm{~K}$ ) in contrast to $0.2 \mathrm{eV}$ at high temperature. Unfortunately, data on $\mathrm{VO}_{x}$ nanotubes are limited. However, ESR measurements on $\mathrm{VO}_{x}$ nanotubes showed similar features to those of $\mathrm{V}_{2} \mathrm{O}_{5}$ single crystals but the inhomogeneous distribution of $\mathrm{V}^{4+}$ was found to increase even more after the hydrothermal treatment. $^{27}$

How these polarons carry current and heat can be derived from the electrical resistivity and thermoelectric power 


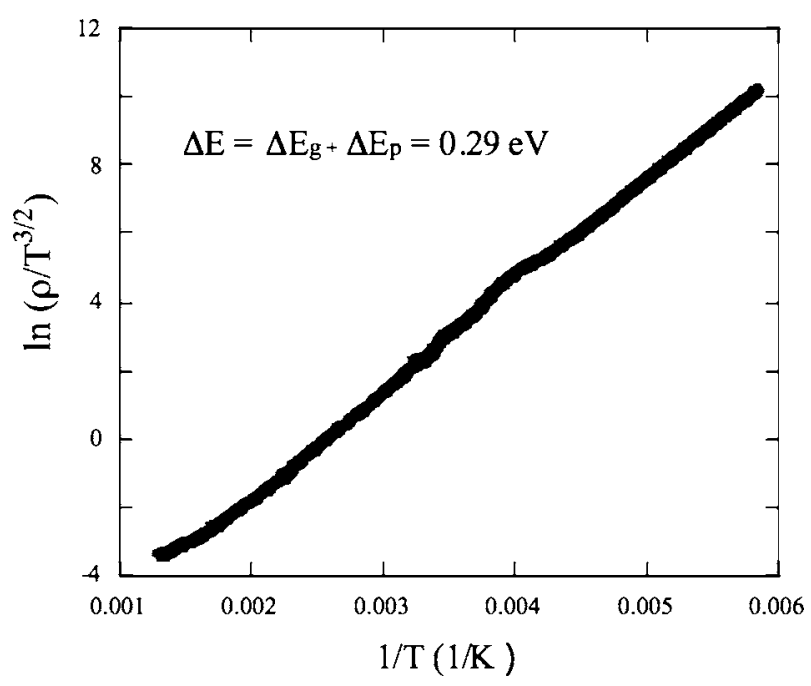

FIG. 3. In order to stress the hopping nature of electrical conduction in $\mathrm{VO}_{x}$ nanotubes, the electrical resistivity is plotted as $\ln \left(\rho / T^{3 / 2}\right)$ vs $1 / T$ for a thick film sample.

measurements. The temperature dependence of the dc resistivity of the nanotubes in a nonadiabatic hopping conductivity can thus be expressed by the formula ${ }^{28}$

$$
\rho=A T^{3 / 2} \exp (\Delta E / k T),
$$

where $\Delta E$ is the activation energy of the conductivity. It can be decomposed into the energies $\Delta E_{g}$ and $\Delta E_{p}$. The first term corresponds to an energy required to put a charge into a polaronic site whereas the second term describes the energy necessary to extract the charge from its polaronic cloud and to propagate it between neighboring sites. These two contributions could be separated if one measures the thermoelectric (Seebeck) coefficient $(S)$. When the conduction happens through hopping of polarons between localized states, although the rate is governed by $\Delta E_{p}$, it does not represent an additional energy gain because the initial and final states have the same energy. Hence, $S$ is determined only by the configurational entropy of these polarons and it gives a temperature independent contribution $\left(k_{B} / e\right) \ln \rho$ (where $\rho$ is the density of charge carriers per site). But if the charges have to be thermally activated into the polaronic state, ${ }^{29}$ similar to the case of a band semiconductor, then $S$ is

$$
S \sim k_{B} / e\left(\Delta E_{g} / 2 k_{B} T\right) .
$$

The resistivity measured in a broad temperature range gives a reasonably good agreement with expression (1) for a nonadiabatic hopping (see Fig. 3). This expression differs from a simple semiconducting activation by the prefactor $T^{3 / 2}$ which comes from the temperature dependence of the hopping frequency of polarons. This term gives a better fit for the data points above $300 \mathrm{~K}$ in Fig. 3. The thermopower data in Fig. 4 follow expression (2), clearly demonstrating that the charge carriers are thermally activated into the polaronic sites. Furthermore, the sign of the Seebeck coefficient indicates predominantly $p$-type conduction. With simple arithmetics we obtain $\Delta E_{g}=0.2 \mathrm{eV}$ and $\Delta E_{p}=0.09 \mathrm{eV}$ energies. This is a reasonable value for polaron binding energy, comparable to $0.1 \mathrm{eV}$ found in manganites. ${ }^{30}$

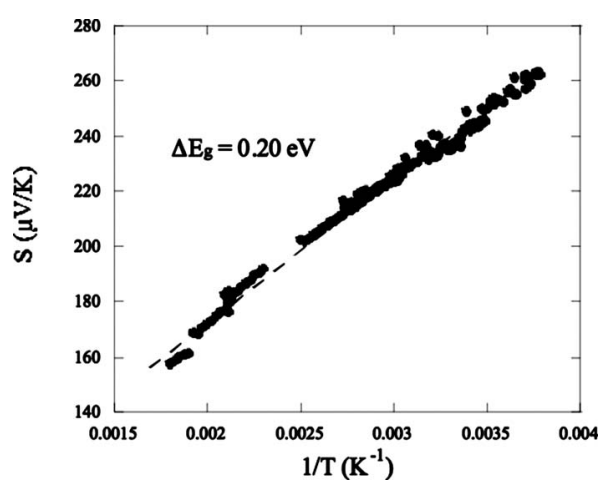

FIG. 4. Thermoelectric power vs inverse temperature measured for a thick film of $\mathrm{VO}_{x}$ nanotubes. The slope gives the activation energy needed for putting charges into the polaronic state.

\section{CONCLUSIONS}

In conclusion, Young's modulus of individual $\mathrm{VO}_{x}$ nanotubes was successfully measured using an AFM technique. $E$ is below $100 \mathrm{GPa}$, which is lower than expected from the bulk value. This is presumably due to the structural defects caused by oxygen vacancies. These vacancies dope the nanotubes, and the charge carriers have a polaronic character. Our electrical resistivity and thermoelectric power measurements revealed that in a macroscopic assembly of nanotubes, the tube-tube contact is good, and despite its porous nature the sample has a reasonable conductance of $0.005 \mathrm{~S} / \mathrm{cm}$. Such a value in combination with respect to their extraordinary structure is promising for battery applications. ${ }^{31}$

\section{ACKNOWLEDGMENTS}

This work was supported by the Swiss National Science Foundation and its NCCR Materials with Novel Electronic Properties (MaNEP).

${ }^{1}$ R. Saito, G. Dresselhaus, and M. S. Dresselhaus, Physical Properties of Carbon Nanotubes (Imperial College Press, London, 1998); in Carbon Nanotubes: Quantum Cylinders of Graphene, edited by R. Saito and A. Zettl (Elsevier, New York, 2008).

${ }^{2}$ C. N. R. Rao and A. Govindaraj, Nanotubes and Nanowires (RSC, Cambridge, 2005).

${ }^{3}$ G. R. Patzke, F. Krumeich, and R. Nesper, Angew. Chem., Int. Ed. 41, 2446 (2002).

${ }^{4}$ F. Krumeich, H.-J. Muhr, M. Niederberger, F. Bieri, B. Schnyder, and R. Nesper, J. Am. Chem. Soc. 121, 8324 (1999).

${ }^{5}$ M. Wörle, F. Krumeich, F. Bieri, H.-J. Muhr, and R. Nesper, Z. Anorg. Allg. Chem. 628, 2778 (2002).

${ }^{6}$ X. Liu, C. Täschner, A. Leonhardt, M. H. Rümmeli, T. Pichler, T. Gemming, B. Büchner, and M. Knupfer, Phys. Rev. B 72, 115407 (2005).

${ }^{7}$ L. Krusin-Elbaum, D. M. Newns, H. Zeng, V. Derycke, J. Z. Sun, and R. Sandstrom, Nature (London) 431, 672 (2004).

${ }^{8}$ P. Poizot, S. Laruelle, S. Grugeon, L. Dupont, and J.-M. Tarascon, Nature (London) 407, 496 (2000).

${ }^{9}$ A. Talledo and C. G. Granqvist, J. Appl. Phys. 77, 4655 (1995).

${ }^{10}$ I. Raible, M. Burghard, U. Schlecht, A. Yasuda, and T. Vossmeyer, Sens. Actuators B 106, 730 (2005).

${ }^{11}$ J.-F. Xu, R. Czerw, S. Webster, D. L. Carroll, J. Ballato, and R. Nesper, Appl. Phys. Lett. 81, 1711 (2002).

${ }^{12}$ M. Niederberger, H.-J. Muhr, F. Krumeich, F. Bieri, D. Günther, and R. Nesper, Chem. Mater. 12, 1995 (2000).

${ }^{13}$ F. Krumeich, H.-J. Muhr, M. Niederberger, F. Bieri, and R. Nesper, Z. Anorg. Allg. Chem. 626, 2208 (2000).

${ }^{14}$ J. P. Salvetat, G. A. D. Briggs, J. M. Bonard, R. R. Basca, A. J. Kulik, T. Stöckli, N. A. Burnham, and L. Forró, Phys. Rev. Lett. 82, 944 (1999).

${ }^{15}$ F. Jachmann and C. Hucho, Solid State Commun. 135, 440 (2005). 
${ }^{16}$ G. Gassner, P. H. Mayrhofer, K. Kutschej, C. Mitterer, and M. Kathrein, Tribol. Lett. 17, 751 (2004).

${ }^{17}$ N. Fateh, G. A. Fontalvo, and C. Mitterer, J. Phys. D: Appl. Phys. 40, 7716 (2007).

${ }^{18}$ J. P. Salvetat, A. J. Kulik, J. M. Bonard, G. A. D. Briggs, T. Stöckli, K. Metenier, S. Bonnamy, F. Béguin, N. B. Burnham, and L. Forró, Adv. Mater. (Weinheim, Ger.) 11, 161 (1999).

${ }^{19}$ B. Lukic, J. W. Seo, E. Couteau, K. Lee, S. Gradečak, R. Berkecz, K. Hernadi, S. Delpeux, T. Cacciaguerra, F. Béguin, A. Fonseca, J. B. Nagy, G. Scányi, A. Kis, A. J. Kulik, and L. Forró, Appl. Phys. A: Mater. Sci. Process. 80, 695 (2005).

${ }^{20}$ S. Hoffmann, F. Östlund, J. Michler, H. J. Fan, M. Zacharias, S. H. Christiansen, and C. Ballif, Nanotechnology 18, 205503 (2007).

${ }^{21}$ I. Hellmann, Ch. Täschner, R. Klingeler, A. Leonhardt, B. Büchner, and M. Knupfer, J. Chem. Phys. 128, 224701 (2008).

${ }^{22}$ V. Eyert and K.-H. Höck, Phys. Rev. B 57, 12727 (1998).
${ }^{23}$ N. Kenny, C. R. Kannewurf, and D. H. Whitmore, J. Phys. Chem. Solids 27, 1237 (1966).

${ }^{24}$ J. Livage, Chem. Mater. 3, 578 (1991).

${ }^{25}$ J. Muster, G. T. Kim, V. Krstic, J. G. Park, Y. W. Park, S. Roth, and M. Burghard, Adv. Mater. (Weinheim, Ger.) 12, 420 (2000).

${ }^{26}$ C. Sanchez, M. Henry, J. C. Grenet, and J. Livage, J. Phys. C 15, 7133 (1982).

${ }^{27}$ K. W. Lee, E. M. Lee, H. Kweon, J. Park, and C. E. Lee, J. Korean Phys. Soc. 49, 1625 (2006).

${ }^{28}$ I. G. Austin and N. F. Mott, Adv. Phys. 18, 41 (1969).

${ }^{29}$ D. Emin, in Electronic and Structural Properties of Amorphous Semiconductors, edited by P. G. Le Comber (Academic, London, 1973).

${ }^{30}$ H. Meskine and S. Satpathy, J. Phys.: Condens. Matter 17, 1889 (2005).

${ }^{31}$ Ch. V. Subba Reddy, S. A. Wicker, E. H. Walker, Q. L. Williams, and R. R. Kalluru, J. Electrochem. Soc. 155, A599 (2008). 